\title{
Electroweak stars: Electroweak Matter Destruction as an Exotic Stellar Engine
}

\author{
Dejan Stojkovic*† \\ SUNY at Buffalo \\ E-mail: [ST/douffalo.edv
}

\begin{abstract}
Stellar evolution from a protostar to neutron star is of one of the best studied subjects in modern astrophysics. Yet, it appears that there is still a lot to learn about the extreme conditions where the fundamental particle physics meets strong gravity regime. After all of the thermonuclear fuel is spent, and after the supernova explosion, but before the remaining mass crosses its own Schwarzschild radius, the temperature of the central core of the star might become higher than the electroweak symmetry restoration temperature. The source of energy, which can at least temporarily balance gravity, are baryon number violating instanton processes which are basically unsuppressed at temperatures above the electroweak scale. We constructed a solution to the Oppenheimer-Volkoff equation which describes such a star. The energy release rate is enormous at the core, but gravitational redshift and the enhanced neutrino interaction cross section at these densities make the energy release rate moderate at the surface of the star. The lifetime of this new quasi-equilibrium can be more than ten million years, which is long enough to represent a new stage in the evolution of a star.
\end{abstract}

35th International Conference of High Energy Physics

July 22-28, 2010

Paris, France

\footnotetext{
* Speaker.

${ }^{\dagger}$ Work done in collaboration with D. Dai, A. Lue and G. Starkman.
} 
It is widely believed that the last stage in the evolution of a star, which is not massive enough to become a black hole, is a neutron star. Stability of a neutron star comes from Fermi pressure of neutrons which balances gravity.

There are some studies arguing the existence of a state between the neutron star and black hole, which is called a quark star. The central density of quark star is higher than the QCD phase transition density $\left[\rho \approx(100 \mathrm{MeV})^{4}\right]$, which allows the transition from the nuclear matter to quark matter. Calculations show that a star containing effectively only three quark flavors $(\mathrm{u}, \mathrm{d}, \mathrm{s})$ can exist in a stable equilibrium where the pressure is provided by the Pauli exclusion principle. However, at higher densities where four or more quark flavors are present, quark matter cannot avoid gravitational collapse.

The question then arises what happens to such a massive star that can not stop at the quark stage? Since in the gravitational collapse matter gets compressed to ever increasing densities and temperatures, eventually the electroweak densities $\left[\rho \approx(100 \mathrm{GeV})^{4}\right]$ will be reached in the central core of the star. At that point electroweak burning can be ignited in which huge amounts of energy can be released. Electroweak burning are basically non-perturbative baryon number violating processes which are essentially unsuppressed above the electroweak scale. In these processes quarks can then be effectively converted into leptons inside the small and dense core of the star. At these densities, the matter is opaque even to neutrinos and the energy released at the center cannot stream freely out of the star. Nevertheless, the energy can be carried out, mostly by neutrinos and antineutrinos. These can also carry out any excess anti-lepton number generated in the electroweak burning, and thus prevent the lepton number chemical potential from halting the consumption of the baryon number. This mechanism can likely provide a stable energy source which can counteract gravity for a while [四].

We separate the electroweak star into three regions, as in Fig. $\square$. The central part of the core is hotter than electroweak symmetry restoration temperature, which is about $100 \mathrm{GeV}$. This region is very dense but small (several $\mathrm{cm}$ ). Since the lepton and baryon numbers are not conserved in this region, baryons are freely transformed to leptons. A $S U(2)$ preserving instanton interaction can convert 9 quarks into 3 leptons, for example:

$$
u d d, c s s, t b b \rightarrow \bar{v}_{e}, \bar{v}_{\mu}, \bar{v}_{\tau}
$$

Since at these temperatures each particle carries about $100 \mathrm{GeV}$ (or more) of energy, this process can release about $300 \mathrm{GeV}$ per neutrino. The typical energy of the produced neutrino is not the only relevant quantity here, we also need the rate at which the energy is released. The sphaleron baryogenesis/baryodestruction rate (above the electroweak symmetry restoration temperature) is proportional to $T^{4}$. However, it turns out that the energy release rate from the core is limited by the quark supply rate (dictated by the gravitational collapse) rather than the maximal electroweak burning rate.

The energy in the form of neutrinos flows out of the central core, and eventually out of the star. However, in the central region, the mean free paths of all particles are short compared to the size of the star. The energy must therefore diffuse out of the central core. Outside the central core, baryon and lepton number are conserved. The density falls with increasing radius, and particle mean free paths increase, however, they are still too short for particles to stream freely out of the star. Eventually, we reach the star's neutrino release shell (i.e. neutrinosphere), where the neutrino 


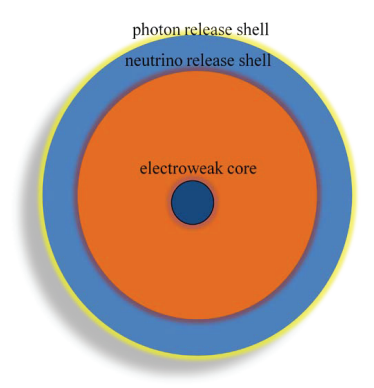

Figure 1: The structure of an electroweak star.

(and anti-neutrino) mean free path is long enough for these particles to flow freely. The neutrinos and anti-neutrinos carry off not just most of the energy produced in the core burning region (the rest is carried off by photons), but also the lepton number, thus permitting the burning to continue. Since the fuel is predominantly baryons not anti-baryons, resulting in the production of an excess of anti-leptons over leptons, there will be a greater flux of anti-neutrinos than neutrinos from the star. This may be a signature of this phase - albeit a rather difficult one to detect. An eventual discovery of anti-neutrino flow from a compact star can be a hint for the electroweak star.

We used the "bag model" [[] as an approximate solution of the region outside the core [四]. The pressure $P$, energy density $\varepsilon$, and number density of particles $n$ can be calculated from the ideal gas distribution using the chemical potential of fermions and without assuming an equation of state a priory. We set the boundary conditions at the center that pressure and energy density are $(100 \mathrm{GeV})^{4}$, and then calculated the structure of the star [四] according to the Tolman-OppenheimerVolkoff equations [B]

$$
\begin{aligned}
& \frac{d P}{d r}=-\frac{(\varepsilon+P)\left(M+4 \pi P r^{3}\right)}{r^{2}(1-2 M / r)}, \quad \frac{d M}{d r}=4 \pi \varepsilon r^{2}, \\
& g_{t t}=\left(1-\frac{2 M_{\text {star }}}{R_{\text {surface }}}\right) e^{-\int_{0}^{P(r)} \frac{2 d P}{P+\varepsilon}}, \quad g_{r r}=\frac{1}{1-\frac{2 M(r)}{r}}
\end{aligned}
$$

where $R_{\text {surface }}$ is radius of the star, while $M_{\text {star }}$ is the mass of the star. Since we are dealing with very dense objects, general relativistic effects described by the metric components $g_{t t}$ and $g_{r r}$ will be very important.

From the numerical solution $P(r), \varepsilon(r)$ and $n(r)$ we learn that the total size of the star is $8.2 \mathrm{~km}$ (radius where pressure and energy density drop to zero) and its mass $1.3 M_{\odot}$ (mass within that radius). The size of the central electroweak core is several $\mathrm{cm}$ (this is the distance at which the density falls below $(100 \mathrm{GeV})^{4}$. Within this central region, the pressure and energy density gradients are very small, so pressure and energy density are practically constant there. In the absence of the active energy source, such a dense star can not be stable and it will start collapsing. However, at some stage, the electroweak interactions are ignited and large quantities of energy are released. While baryon and lepton number violating interactions are spending the quark fuel, gravitational collapse is bringing more material to the central part of the core where electroweak symmetry is restored. Once the amounts of the released and incoming energies are the same, the star enters a quasi-stable state that can last for a while. 
Most of the neutrinos near the central region have very high energies. Since their mean free path in this region is very short, they can not escape from the star. However, at lower energies and lower densities they can. We define the neutrino escape radius as the distance form the center at which a neutrino with a certain energy can escape from the star. The radius of the neutrino release shell is thus just the neutrino escape radius. A very important quantity in this context is the neutrino mean free path which depends on the cross section of neutrino scattering $\sigma$, i.e. $1 / \lambda=\sum_{i} \sigma_{i} n_{i}$.

Unlike ordinary stars, particles propagating through the electroweak stars suffer large gravitational redshift. We found that a particle retains only a fraction of $10^{-3}$ of its original energy near the center. We then estimated the maximal energy release rate at the surface of such a star. The maximal energy release rate can be obtained from the free fall time of the incoming quark matter into the electroweak core. We obtained the numerical value of about $10^{27} \mathrm{MeV}^{2}$. It is important to compare this rate with the electroweak baryogenesis/baryodestruction interaction rate which is about $10^{34} \mathrm{MeV}^{2}$. Since the baryodestruction rate is much larger than the maximal energy release rate, we can reasonably assume that when particles reach the electroweak core, they decay into neutrinos instantaneously. Otherwise, the infalling matter would pile up and form a black hole. This also implies that the released energy from the star does not depend on the precise details of the electroweak burning since the energy release rate from the core is limited by the quark supply rate (dictated by the gravitational collapse) rather than the maximal electroweak burning rate.

We then calculated the energy release rate at infinity more accurately taking into account the transport of energy through the star and the relativistic effects [四] using the equation

$$
4 \pi r^{2} \lambda \frac{d\left(S(r) g_{t t}(r)\right)}{\sqrt{-g_{r r}(r)} d r}=-L .
$$

Here $S(r) \equiv d E(r) /(d t d A)$ is the energy flux at the radius $r, A$ is the area, while $L \equiv d E / d t$ is the energy release rate at infinity (which is about the same order as the intensity at the neutrinosphere). The factor $g_{t t}(r)$ describes both the redshift and time delay. The factor $g_{r r}(r)$ takes into account that the space-time inside the star is not flat. The energy flux $S(r)$ can be found from the energy density knowing the energy (i.e. chemical potential) of neutrinos. The metric coefficients are given by Eq. (DI). It is then straightforward to calculate the energy release rate at infinity, i.e. $L$, given all the other parameters.

For the original energy of the neutrinos of $300 \mathrm{GeV}$, we find that the radius of the neutrino release shell must be less than $8.1 \mathrm{~km}$. With this maximal number, the energy release rate is about $10^{24} \mathrm{MeV}^{2}$. Integrating this, we find that it takes about $10^{15} \mathrm{sec}$ (10 million years) to release energy equivalent of $1 M_{\odot}$. This is the minimal life-time of the electroweak star, provided that the electroweak burning does not stop before all the quark fuel is spent. This is long enough to be considered as a new phase in evolution of very massive stars.

\section{References}

[1] D. C. Dai, A. Lue, G. Starkman and D. Stojkovic, arXiv:0912.0520 [hep-ph], To appear in JCAP

[2] A. Chodos, R. L. Jaffe, K. Johnson, C. B. Thorn and V. F. Weisskopf, Phys. Rev. D 9, 3471 (1974).

[3] J. R. Oppenheimer and G. M. Volkoff, Phys. Rev. 55, 374 (1939). 